\title{
Information Requirements Engineering for Data Warehouse Systems
}

\author{
Robert Winter \\ Institute of Information Management \\ University of St. Gallen \\ Mueller-Friedberg-Strasse 8 \\ St. Gallen, Switzerland \\ +41712242935 \\ Robert.Winter@unisg.ch
}

\author{
Bernhard Strauch \\ LGT Financial Services \\ Herrengasse 13 \\ Vaduz, Principality of Liechtenstein \\ +4232352301 \\ Bernhard.Strauch@lgt.com
}

\begin{abstract}
Information requirements analysis for data warehouse systems differs significantly from requirements analysis for conventional information systems. Based on interviews with project managers and information systems managers, requirements for a methodological support of information requirements analysis for data warehouse systems are derived. Existing approaches are reviewed with regard to these requirements. Using the method engineering approach, a comprehensive methodology that supports the entire process of determining information requirements of data warehouse users, matching information requirements with actual information supply, evaluating and homogenizing resulting information requirements, establishing priorities for unsatisfied information requirements, and formally specifying the results as a basis for subsequent phases of the data warehouse development (sub)project has been proposed. The most important sources for methodology components were four in-depth case studies of information requirements analysis practices observed in data warehousing development projects of large organizations. In this paper, these case studies are presented and the resulting consolidated methodology is summarized. While an application of the proposed methodology in its entirety is still outstanding, its components have been successfully applied in actual data warehouse development projects.
\end{abstract}

\section{Categories and Subject Descriptors}

D.2.1 [Requirements/Specifications]: Elicitation Methods, Methodologies.

\section{General Terms}

Design.

\section{Keywords}

Data warehouse systems; Requirements specification; Method engineering.

Permission to make digital or hard copies of all or part of this work for personal or classroom use is granted without fee provided that copies are not made or distributed for profit or commercial advantage, and that copies bear this notice and the full citation on the first page. To copy otherwise, to republish, to post on servers or to redistribute to lists, requires prior specific permission and / or a fee.

SAC'04, March 14-17, 2004, Nicosia, Cyprus

Copyright 2004 ACM1-58113-812-1/03/04_..\$5.00

\section{INTRODUCTION}

In contrast to conventional information systems, data warehouse systems are developed incrementally over a long time period and involving many organizational units. As a consequence, information requirements are changing in time and maybe ambiguous. Data warehouse systems are developed to support decision makers by providing consistent, timely, subject oriented information at the required level of detail. But information requirements are difficult to specify due to the uniqueness and / or missing structure of many decision processes, the reluctance of decision makers to disclose process details, the inherent future orientation of this kind of information supply and conceptual inconsistencies within large organizations [16].

In actual data warehouse system development projects, it is therefore extremely challenging to elicit, specify and homogenize information requirements. As a consequence, data warehouse systems are sometimes developed based on an incomplete and / or inconsistent set of information requirements - a fact that is related to the failure of these projects [12]. In order to support project managers in eliciting, specifying and homogenizing information requirements for data warehouse systems, a dedicated method is called for. In contrast to existing requirements engineering approaches that aim at existing, well understood requirements, this method has to take into account that information requirements of decision makers refer - at least partially - to information that does not necessarily exist presently, but that can be derived from existing sources and components [6].

In this paper, a requirements engineering method for data warehouse systems is proposed. The paper focuses on four case studies which represent successful business practices for information requirements analysis, thereby complementing the method description in [16]. Expert requirements for data warehouse information requirements analysis are summarized in section 2 . Section 3 is a short review of related work in the light of these requirements. The method engineering approach is summarized in section 4. Being the primary sources of components (e.g. activities, document types, modeling techniques) for the proposed method, four case studies of development projects in large Swiss companies are presented in section 5 . The proposed method is outlined in section 6 . 


\section{EXPERT REQUIREMENTS FOR DATA WAREHOUSE INFORMATION RE- QUIREMENTS ANALYSIS}

By interviewing data warehouse project managers from various large Swiss and German companies, the following requirements for an effective methodological support of the information requirements analysis phase in data warehousing projects have been identified [15]:

1. Multi-stage, hierarchical approach: To avoid wasting resources and guide the specification process, the matching of information demand and information supply should start on an aggregate level and should be refined later.

2. 'Information map': As one of the results of the information requirements analysis, a document should be created that represents where data are sourced from, which organizational units use which data, which data relate to which concepts, which concepts are homonyms or synonyms, etc. on an aggregate level.

3. 'As is' information provision: It must be documented (and is a necessary input for creating the information map) which source systems provide which data in which quality.

4. 'To be' information state: By analyzing actual and projected overlaps of information supply and information demand, the 'to be' information state is planned to meet not only actual, but also projected information requirements.

5. Assignment of priorities: Since limited resources allow only selected unsatisfied information requirements to be covered by the data warehouse system, evaluation criteria have to be developed and coordinated which then allow for deriving priorities.

6. Homogenization of concepts: Data from different operational sources can only be integrated if the underlying semantic concepts have been homogenized.

7. Integration with subsequent development phases: In order to create results that are reusable in subsequent phases of the data warehouse development project, models for information requirements representation should be aligned with - or even be identical with - models used for data warehouse design and / or data mart design. Therefore project managers prefer conceptual models for multidimensional data.

8. Documentation of meta data: Information requirements analysis creates valuable meta data that should be directly transferable into the meta data management system instead of having to be manually recorded and integrated in subsequent phases of the data warehouse development project.

The proposed method is designed to meet these requirements and to support the systematic specification of current as well as future information requirements.

\section{RELATED WORK}

A large number of approaches to information requirements analysis have been developed both by academia and companies / consultants. Back in 1995, Beiersdorf describes not less than 28 techniques and technique combinations for information requirements analysis [1]. Most of these techniques, however, have been developed for supporting the development of conventional information systems and are therefore only partly suitable for data warehousing projects.

Like Business Systems Planning [11], Information Engineering [5] or the method of critical success factors [13], most methods combine specific elements with basic components like interviews, questionnaires and job analyses which of course are also applicable for data warehouse development projects. But as a whole, they do not meet the requirements described in section 4 sufficiently (for a detailed discussion see [15], pp.77ff).

Although it seems to be obvious that matching information requirements of future data warehouse users with available information supply is the central issue of data warehouse development, only few approaches seem to address this issue specifically. Based on whether information demand or information supply is guiding the matching process, demand driven approaches and supply driven approaches can be differentiated:

- Demand driven approaches are aimed to determine information requirements of data warehouse users. According to Hansen [10, p.319], end users alone are able to define the business goals of the data warehouse systems correctly so that end users should be enabled to specify information requirements by themselves.

However, end users are not capable to specify their objective, unsatisfied information requirements because their view is subjective by definition, because they cannot have sufficient knowledge of all available information sources, and because they use only a business unit specific interpretation of data $[15, \mathrm{p} .115]$. Moreover, end users can often not imagine which novel information the data warehouse system could supply [4, p.7] [6, p.55].

- Supply driven approaches start with an analysis of transactional source systems in order to reengineer their logical data schemas. By selecting items from the consolidated data schema, information requirements can then be specified by end users. The data schema subset can directly reused as input for the data warehouse data schema.

However, these approaches risk to waste resources by handling many unneeded information structures. Moreover, it may not be possible to motivate end users sufficiently to participate because they are not used to work with large data models developed for and by specialists [6, p.55].

A special type of demand driven approaches is to derive information requirements by analyzing business processes in increasing detail and transform relevant data structures of business processes into data structures of the data warehouse [2]. The data warehouse systems regarded in our research have been developed to support exclusively decision processes. For decision processes, however, a detailed business process analysis is not feasible because the respective tasks are often unique and unstructured or, what is even more important, because decision makers / knowledge workers often refuse to disclose their processes in detail.

\section{METHOD ENGINEERING APPROACH}

The first expert requirement (see section 2) calls for an approach that is guided by information demand instead of information supply. Only by guiding requirements analysis by what is actually 
needed (instead of what is available), the risk of wasting resources is minimized [15]. Since the business process oriented approach is not applicable if the data warehouse system has to support decision / knowledge processes, we focus on a 'conventional' demand driven approach. Of course the proposed method should overcome the shortcomings listed in section 3, i.e. a multi-stage approach has to be taken, users have to be supported in specifying objective (and not subjective) information demands, novel information (e.g. information from sources that users might not be aware of) has to be included, the homogenization of information requirements has to be supported, selection and priority assignment mechanisms have to be provided for project management, and aggregate as well as semi-formal specification documents have to be created.

Method engineering is the discipline of combining an activity model (comprising activities, activity structure, and activity sequence), a role model (comprising types of responsibilities and ownership), certain modeling techniques, certain types of documents and probably certain tools (that support a modeling technique) into a consistent structure [3]. Figure 1 illustrates the meta model of a such a method.

Activities, roles, modeling techniques and types of document are usually adopted from successful business practices or from related academic work. Based on a conceptual analysis of these components and method design requirements (e.g. those summarized in the preceding paragraph), potential components are evaluated and selected components are integrated into a consistent method. An important portion of the method engineering process is the analysis of case studies as a base for selecting appropriate method components (e.g. activities, modeling techniques, information objects, document types).

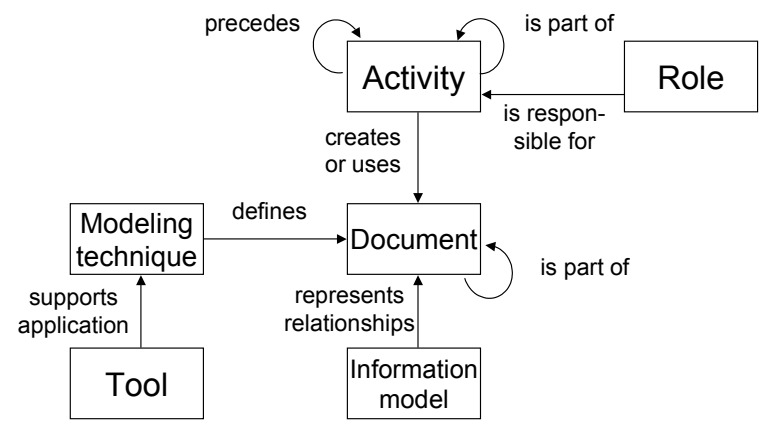

Figure 1. Method meta model

\section{CASE STUDIES}

The following four case studies were used to derive all important components of a method for information requirements analysis for data warehouse systems.

\subsection{Case A: Business unit 'operations' at UBS Switzerland}

UBS is the largest bank in Switzerland and also one of the largest banks in the world. Being a universal bank, UBS is active in retail banking, investment banking, private wealth management, institutional asset management, securities trading, etc. UBS' business unit 'operations' in Switzerland is responsible for processing all payments, processing securities transactions and securities custody for all Swiss and selected international UBS units.
The case study summarizes the development of a data warehousing infrastructure from the viewpoint of information requirements analysis. The development project comprises not only the central data warehouse layer, but also the development of data marts to support decision processes in the business unit 'operations'.

Since more than 60 transactional systems were considered as potential data sources for the data warehouse, a demand-driven approach was chosen. Information requirements as well as the quality of current information supply were elicited from decision makers from the affected departments custody and payments by means of a questionnaire. This survey was done with ca. 180 decision makers on three hierarchy levels (team leader, department manager, vice president) during six months in 1997. The results were stored in a data base to serve as a foundation for subsequent analyses.

The requirements catalogue derived from that database turned out to be too voluminous. In addition, the quality of specifications was insufficient for data mart design purposes. Although representing important information requirements on an aggregate level, the survey results were too superficial to be usable as requirements in the data warehouse system development process. Moreover, it was not clear which requirements could be realistically met using actual information systems.

The following conclusions were drawn for future information requirements surveys:

- Questionnaires alone are insufficient and have to be complemented by interviews.

- Interviews have to be conducted by specialists from the respective department.

- $\quad$ Survey participants should be asked to prepare the interviews by analyzing their current and future decision processes.

- It must be avoided to produce (high) expectations regarding the decision support made available by the developed system.

Based on these findings, the next steps in structuring and homogenizing information requirements at UBS were as follows:

- Identification of targeted users and dominant applications: In order to reduce the amount and complexity of information requirements to be dealt with, targeted users as well as dominant applications must be identified.

- Iterative specification and priority assignment: In several workshops with targeted users (decision makers), information requirements were iteratively refined until a level of detail was reached that allowed for a derivation of conceptual data mart models.

- Consolidation of heterogeneous information requirements: Information requirements of different groups of targeted users can be expected to be at least partially inconsistent. As long as the development project does not affect multiple business units, however, it should be possible to consolidate information requirements by agreeing on a common terminology, common dimension hierarchies, etc. It proved useful to maintain inconsistent classifications, conflicting definitions etc. by marking them to be department-specific and making explicit their relationship to common structures and terms. 
- Analysis of data sources: Before specified information requirements are fed into the subsequent stages of data warehouse development, a preliminary analysis of data availability should be carried out. In this project, only about $50 \%$ of the information requirements could be realistically met.

- Review priority assignments: Since many information requirements cannot be met due to unavailability of data sources, users must be given the chance to re-prioritize their requirements.

The following conclusions were drawn for future projects:

- A complete compilation of information requirements by survey was considered to create too much irrelevant information, to involve too many people and to be generally too expensive. Instead, targeted interactive surveys should focus on certain user groups and involve information systems specialists from the respective functional / process field.

- In particular, expectations at the system in development must be managed very carefully. Asking every decision maker which information she/he would like to have is likely to cause disappointment and jeopardize the development project success because a high portion of information requirements can simply not met by the current information systems.

- If requirements catalogues are used at all, they should reflect as-is rather than to-be information supply.

- Small, incremental data mart projects are considered as an efficient way to iteratively elicit more future-oriented information requirements while not raising too high expectations too early.

\subsection{Case B: Project 'MIS' at UBS Switzerland}

This case study is also originated at the business unit which is responsible for payments and custody processing, mainly for other Swiss UBS units. In contrast to case A, this case represents the enhancement of an existing analytical information system. This 'MIS payments' information system is based on the enterprise data warehouse and some business unit-specific data marts. While in production, the MIS payments system satisfied about $80 \%$ of all specified information requirements. Unsatisfied information requirements as well as informal requests by key users were collected by the 'system owner', i.e. the IS manager in charge for the MIS payments system.

The case describes the process of enhancing 'MIS payments' functionality by adding new data sources. Information requirements for this project were specified as follows:

- Analysis: The as-is information supply by the MIS payments system was compiled into a catalogue and was sent to users. Together with that catalogue, a questionnaire asked users to check which information was still needed and to specify which additional information was requested. Regarding additional information, users had to specify aggregation level, periodicity, source systems and reference values.

Business users were not willing to supply the requested specifications because they perceived the questionnaire as being to technical. In contrast, unstructured and informal collection of requirements by the IS management was considered to be appropriate.
- Homogenization of terms: Being a kind of mediator between different groups of users, the MIS payments systems owner created an ontology that included various synonyms and homonyms in use across the business unit.

- Assignment of priorities: The priority assignment process was based on the questionnaire analysis: The more users requested a specific information, the higher priority was assigned to the respective information requirement. A second source of priority assignments was the importance that users were able to assign to specific information subjects in the questionnaire.

- $\quad$ Source systems: The MIS payments system owner estimated that only about $10 \%$ of new information requirements could not be satisfied by existing operational information systems in sufficient (data) quality. As a consequence, an expensive data quality evaluation of source systems was omitted.

- Conceptual modeling: Conceptual modeling of the resulting, extended information model of MIS payments was performed by specialists from an IT unit (and not by the business unit owning the system).

The following conclusions were drawn for future projects:

- In order to avoid exaggerated expectations of users regarding the system extension, it was clearly communicated at all times that the upgrade is incrementally and that new requirements may be supported not completely.

- Even experienced users experienced problems when being asked to specify their information requirements 'technically' (i.e. including aggregation level, periodicity, data sources, etc.). As a consequence, users were asked to formulate typical 'business questions' that represent information requirements informally and implicitly. Based on the posted business questions, the explication process was then performed by the IS management.

- By using the existing MIS and by being encouraged to use novel OLAP applications, users were encouraged to define new information requirements by posting new business questions.

\subsection{Case C: Logistics at Swiss Armed Services}

The performance of modern armed forces significantly depends on effective / efficient logistics and respective information systems. Such information systems must provide military as well as management information. Within the context of a comprehensive redesign of the Swiss Armed Services, the development of a data warehousing strategy and the design of a data warehouse infrastructure are important issues [9].

SISLOG, the strategic logistics information system of Swiss Armed Forces is intended to enable commanders to assess the entirety of available resources regarding identification, status, personnel, costs, etc. [14, p.2]. SISLOG is being implemented as information, planning and simulation system based on a data warehouse. The system shall provide information not only related to military, but also related to 'business' questions, e.g.:

- Which costs are entailed by a given level of preparedness?

- Which level of preparedness can be achieved? 
- Which logistics services can be performed based on a given budget?

In order to specify information requirements for SISLOG, interviews have been carried out with key users. These specifications were however on a high level of abstraction so that no conceptual schema could be derived. For each high-level unit, workshops were used to refine respective information requirements. 'Business questions' were formulated as detailed as possible, e.g. by looking at actual capacity requirements for specific types of equipment and specific types of operations. The most important output of these workshops has been a set of detailed calculation rules which were used to derive capacity supply / demand estimations. The following conclusions were drawn for future projects:

- Several requirements (such as multilingual capabilities, channel-specific capabilities) were made during the interviews that could not be considered as information requirements.

- Although it is necessary to prepare users for information requirements elicitation interviews, preparation must not go so far that the creativity of users is significantly restricted. The tradeoff between creativity and preparation is however not sufficiently understood. In some interviews where user creativity was considered to be very important, preparation work was so limited that no significant results were achieved.

- In order to conduct interviews efficiently, the type of results must be specified. Whether informal 'business questions' are to be collected or whether a conceptual information schema is to be constructed makes a big difference for the preparation and realization of interviews.

- If interviews have to be complemented by workshops due to the unsatisfactory quality of interview results, significant resources have to be made available. For all important highlevel units, workshops had to be conducted that involved all important decision makers in order to produce consistent, reliable results.

- In a situation where a large number of heterogeneous information systems is involved, an important problem of requirements specification arises from the fact that decision makers and even IS owners do not exactly know which system is the leading system for which data. In addition, data quality issues can not be resolved satisfactory if IS architectures are complex and / or designed to implement a certain amount of redundancy.

\subsection{Case D: Project 'Reports analysis' at Swiss Life}

Swiss Life is the largest life insurance company in Switzerland. A data warehouse system is operational at Swiss Life since 1997. Its most important applications and experience from its design and development are documented in [7] and [8]. The focus of this case study is the 'aggregate information map', a specific modeling technique (and document type) that has been developed and used at Swiss Life for supporting several data warehouse extension projects [15, pp. $120 \mathrm{ff}$.].

In several departments of the 'individual contracts' business unit of Swiss Life, numerous reports are created using data directly drawn from operational information systems. No department has an overview regarding all the reports in use. On the one hand, a large amount of redundancy may be expected. On the other hand, problems arise when different departments create similar reports with inconsistent results because the information is derived from different systems and / or by derived by different procedures.

If a common data warehouse served as a 'single point of truth' for all reporting purposes in that business unit, it was assumed that both types of problems could be avoided and that significant cost savings could be realized over a medium planning horizon. In order to substantiate these expectations, the project 'reports analysis' was initiated.

By means of a questionnaire, information system owners were asked to document all reports that were created using data of the respective system. Questionnaire data then were collected in a database which was build specifically for this survey. For every report, it had to be specified which figures were included in that report (e.g. amount of claims per period per sales region), how often the report is generated, which information system is used to source data, which persons receive the report, etc. In a subsequent step, the 'reports analysis' project manager collected potentially synonymous, potentially homonymous, and even similar figures (e.g. gross premium and net premium) in so-called concept clusters. Eventually more than 600 figures were arranged into 17 concept clusters, thereby creating an 'aggregate information map'.

Based on the aggregate information map, it was systematically tested whether potentially homonymous figures were used in several reports. An analysis of the data sources and derivation rules in the respective reports then proved whether the regarded figures had to be treated as homonyms (i.e. new terms had to be introduced or terms had to be renamed) or whether the same figure was derived from the same source data in the same way - which was rather the exception than the rule.

The aggregate information map was also used to document which information systems were involved in certain activities (e.g. premiums calculation) or which departments generally use which types of information. By that means, an aggregate overview of all reporting activities within the business unit 'individual contracts' could be created.

While the aggregate information map allows only broad, aggregate analyses, the contents of the report analysis database allow for specific queries, e.g. which figures are derived from which source data, which departments use which figures, which persons requested which figures or which IS owner is indirectly responsible for which figures (an important question for data quality management).

Though being useful to identify inconsistencies and redundancies in reporting structures, the reports analysis project only addressed to 'production side' of information logistics. If information requirements analysis is understood to be demand-oriented, this project had to be expanded to include (report) users and help them to locate new information to support their decisions. This expansion however was considered to be too expensive and timeconsuming.

An interesting side effect of the reports analysis project was that with the exception of very few cases - information flows were found to be quite straightforward from source systems into reports so that a data warehouse's benefits as 'single version of truth' 
were quite insignificant for the respective business unit. It was therefore decided for most reports not to resource there data from the data warehouse. If reports are to be extended or if new reports are to be developed, however, it has to be checked whether data should be sourced from the data warehouse or directly from a source system.

\section{PROPOSED METHOD}

When comparing the expert requirements for a method from section 2 with the case study descriptions in section 5 , it becomes evident that no observed practices met the complete set of requirements. The level of contribution of the four case studies ' $A$ ', ' $B$ ', ' $C$ ' and ' $D$ ' as well as their level of detail is illustrated in figure 2.

The companies however developed successful 'partial' solutions, i.e. they developed certain activities that can be compiled into a method that, as a whole, satisfies the expert requirements from section 2. Let 'A1'..'A11' denote eleven activities observed in case study ' $A$ ' that were assessed to be relevant to the proposed method. 'B1'..'B8' denote eight relevant activities observed in case study 'B', respectively. Similarly, ' $\mathrm{C} 1$ '..' $\mathrm{C} 8$ ' denote eight relevant activities observed in case study ' $C$ ', and 'D1'..'D4' denote respective activities observed in case study ' $D$ '. When being evaluated with regard to the eight expert requirements from section 2, the selected activities observed in the four case studies performed as illustrated in figure 3 (for evaluation details see [15]). It becomes evident that the practices described in the four case studies are somewhat complementary with regard to the expert requirements. Hence, a combination of the best performing activities can be assumed to be a reasonable first start for a comprehensive method. When a specific activity did not meet expert requirements completely, it was combined with similar activities observed in other case studies or complemented by practices documented in related work.

A common, consistent sequence of similar activities was observed in most cases that includes two different supply-demand matching steps: After some initialization activities, information requirements for regarded decision processes were first matched with available information on an aggregate level. Based on that aggregate match, requirements then were brought into a priority sequence, the most important requirements being elaborated in more detail and then homogenized. A second matching activity then was used to synchronize information supply and information demand on a full level of detail. In a final modeling phase, information requirements (and appropriate meta data) is transformed into a format that can be reused in subsequent development phases.
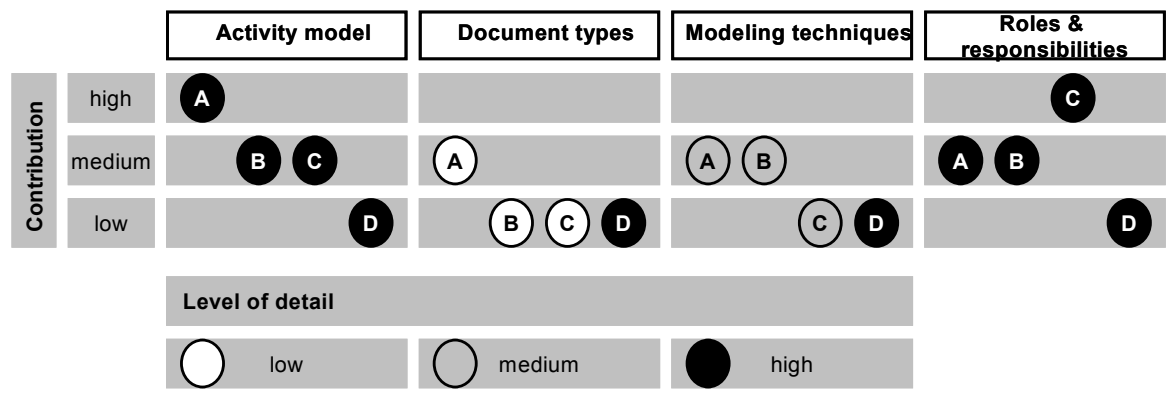

Figure 2. Positioning of the case studies
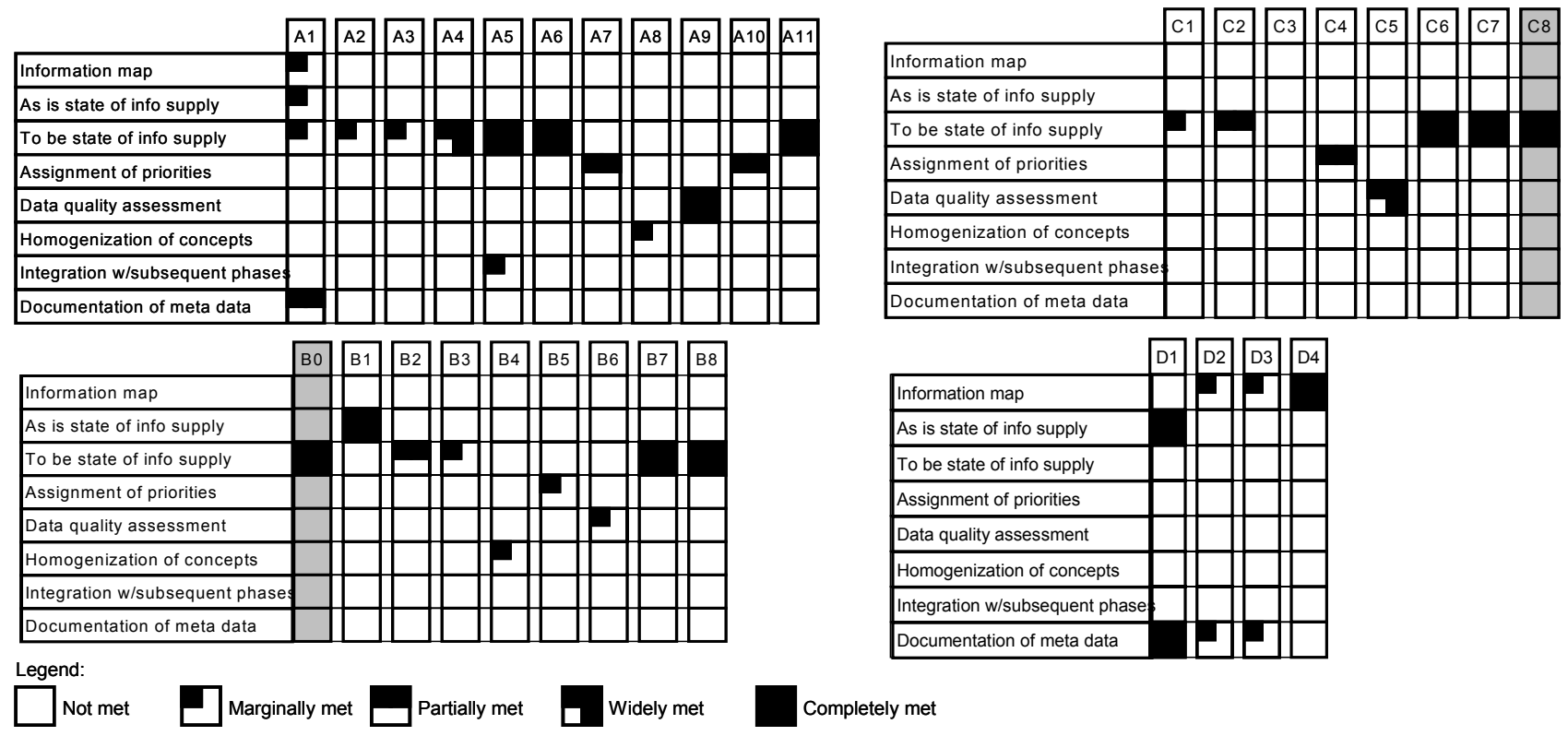

Figure 3. Contribution of the case studies to the method requirements 
The resulting consolidated activity model is illustrated by figure 4. A detailed description of the activities can be found in [16]. All proposed document types, modeling methods as well as the role model and the information model of the method are described in [15].

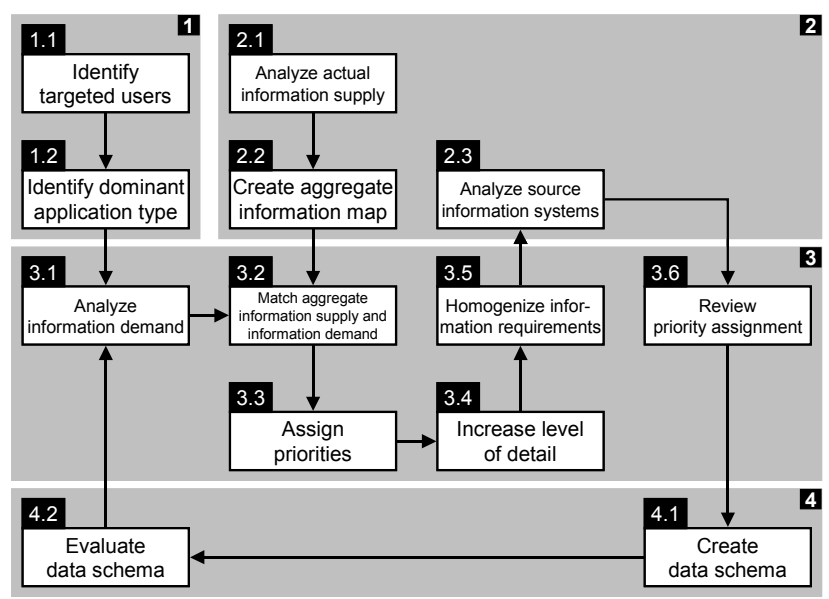

Figure 4. Consolidated activity model

\section{CONCLUSIONS}

Based on a discussion of the specific nature of information requirements analysis for data warehouse systems, a set of practitioner requirements for methodological support for this important problem has been presented. Since related work provides no consistent methodology that meets these requirements, we presented four case studies of successful business practices and summarized the process of combining, homogenizing, complementing and sequencing these practices into a comprehensive method. The resulting demand-oriented method supports an iterative, priority oriented approach to eliciting, documenting, visualizing, homogenizing and specifying information requirements. As primary deliverables, a conceptual, multi-dimensional data schema and a large amount of meta data are created.

From a conceptual viewpoint, requirements analysis is made consistent with incremental data warehousing development practices by the proposed approach. Information demand and information supply are synchronized in a two-step, hierarchical procedure as requested by most experts. End-user involvement is encouraged by using business questions for requirements documentation and discussion instead of formal models. Semantic inconsistencies in large, decentralized organizations are addressed, and priority assignments are supported.

Even if the method has not been applied in its entirety [15], large portions of it have been developed together with companies and have been directly applied in the context of the presented cases. Significant reuse of requirements meta data has been observed as well as increased manageability and better documentation of the entire 'requirements analysis' phase as part of large data warehouse development projects.

An important open issue is the contradiction of proposing a comprehensive method and experiencing that in most companies, corporate standards and existing tools (or models or skills) limit the applicability of new methods significantly. Moreover, a sophisticated method application requires some infrastructure (e.g. for meta data management) that seems to be increasingly problematic to maintain in the light of the tendency to organize data warehouse development as a business-driven project in decentralized organizations.

\section{REFERENCES}

[1] Beiersdorf, H.: Informationsbedarf und Informationsbedarfsermittlung im Problemlösungsprozess „Strategische Unternehmungsplanung“ (vol. 5). Hampp: München, Mering 1995.

[2] Böhnlein, M., Ulbrich-vom Ende, A.: Business Process Oriented Development of Data Warehouse Structures, in: Jung, R., Winter, R. (Eds.): Data Warehousing 2000 - Methoden, Anwendungen, Strategien; Physica: Heidelberg 2000, 3-21.

[3] Brinkkemper, S., Lyytinen K. and Welke R.: Method Engineering, Chapman \& Hall, London 1996.

[4] Connelly, R. A., McNeill, R., Mosimann, R. P.: The Multidimensional Manager. Cognos Inc.: Ottawa 1999.

[5] Finkelstein, C.: An Introduction to Information Engineering. Addison Wesley: Sydney etc. 1989.

[6] Gardner, S.: Building the Data Warehouse. Communications of the ACM, 41 (1998), 9, 52-60.

[7] Garzotto, A.: MASY - Ein Praxisbericht; Informatik / Informatique, 1999, 1, 16-19.

[8] Garzotto, A.: MASY - Ein Erfahrungsbericht zum Thema Data Warehouse, in: Jung, R., Winter, R. (Eds.): Data Warehousing Strategie. Springer: Berlin etc. 2000, 161-167.

[9] Grütter, G.: Data Warehouse-Strategie in der Logistik der Schweizer Armee, in: Jung, R., Winter, R. (Eds.): Data Warehousing Strategie. Springer: Berlin etc. 2000, 181-195.

[10] Hansen, W.-R.: Vorgehensmodell zur Entwicklung einer Data Warehouse-Lösung, in: Mucksch, H., Behme, W. (Eds.): Das Data Warehouse-Konzept. 2nd ed.; Gabler: Wiesbaden 1997, 311-328.

[11] IBM Corp.: Business Systems Planning - Information Systems Planning Guide. 4th ed., IBM-Form GE20-0527-4, Atlanta 1984

[12] List, B., Schiefer, J., Tjoa, A. M.: Use Case Driven Requirements Analysis for Data Warehouse Systems, in: Jung, R., Winter, R. (Eds.): Data Warehousing 2000 - Methoden, Anwendungen, Strategien; Physica: Heidelberg 2000, 23-39.

[13] Rockart, J. F.: Chief executives define their own data needs; Harvard Business Review, 57 (1979) 2, 81-93.

[14] Schneider, D. H.: Führung \& Darstellung der Logistik im 21. Jahrhundert, http://www.vbs.admin.ch/internet/GST/ Uglog/ d/projekte/IMAGES/it_logistikXXI. pdf, downloaded 200103-20.

[15] Strauch, B.: Entwicklung einer Methode für die Informationsbedarfsanalyse im Data Warehousing. Doctoral Thesis. University of St. Gallen, 2002.

[16] Winter, R.; Strauch, B.: A Method for Demand-driven Information Requirements Analysis in Data Warehousing Projects. Proceedings of the 36th Hawaii International Conference on System Sciences, IEEE 2003 